\title{
Left renal artery thrombosis in a 78-year-old patient with atrial fibrillation
}

\author{
Bilgehan Erkut* and Azman Ates \\ Department of Cardiovascular Surgery, Atatürk University, Medical Faculty, Erzurum, Turkey
}

\begin{abstract}
Renal artery thrombosis is a rare clinical condition. Often, there is a delay in diagnosis due to non-specific clinical complaints. We presented a woman with an elderly atrial fibrillation who developed thrombosis of the left renal artery. Despite the anticoagulant treatment given, patient did not benefit and the kidney was fully damaged.
\end{abstract}

\section{Brief Report}

A 78-year-old woman with secondary mitral and tricuspid valve regurgitation and atrial fibrillation was referred to the emergency department with complaint of sudden and persistent pain in the left abdominal cavity, starting 2 days ago. Physical examination revealed tenderness and side pain in the left abdomen. The patient was treated due to chronic obstructive pulmonary disease. He was febrile and blood pressure was $140 / 90 \mathrm{mmHg}$. Pulse rate was $120 / \mathrm{min}$ and arrhythmia. The blood oxygen saturation was $88 \%$, PO2 value was 55 $\mathrm{mmHg}$. Laboratory tests revealed a white blood cell count of 14.200, a blood urea nitrogen level of $44 \mathrm{mg} / \mathrm{dL}$, a serum creatinine level of $1.4 \mathrm{mg} / \mathrm{dL}$. LDH and CRP values were above normal limits. Hematuria and proteinuria were detected in urine analysis. A transesophageal echocardiogram was performed and a thrombus in the left atrial was demonstrated (Figure 1). An ischemic event of embolic origin was considered as the cause of abdominal pain and abdominal tomography was done. Abdominal computerized tomography (CT) showed that the left renal artery was obstructed by thrombus (Figure 2). Because it is an embolic thrombus, anticoagulation treatment was started by subcutaneous route (Innohep, Abdi Ibrahim, Turkey; SC 100IU/kg/ day), immediately. Nausea, vomiting and pain decreased after 2 days of treatment. Serum creatinine level dropped to $1.2 \mathrm{mg} / \mathrm{dL}$. After 2 days, CT was repeated, and seen that the left renal artery thrombosis and infarction continuing and left renal nonfunctioning (Figure 3). There was no improvement in renal perfusion despite the anticoagulant treatment. The patient was considered a delayed case and no other interventional treatment was needed.

Most of the renal artery thrombosis can be caused by atrial fibrillation, aortic interventional procedures, abdominal trauma, renal surgery, and blood clotting disorders [1]. Because the duration of kidney ischemia is 1-6 hours, the diagnosis of renal artery thrombosis should be made as soon as possible in order to preserve kidney function. Therefore, in patients with side pain that cannot be explained for other reasons, renal artery thrombosis diagnosis should be remembered and contrast CT should be performed as soon as possible. In addition to CT imaging, magnetic resonance imaging can also be used to confirm the diagnosis $[1,2]$.

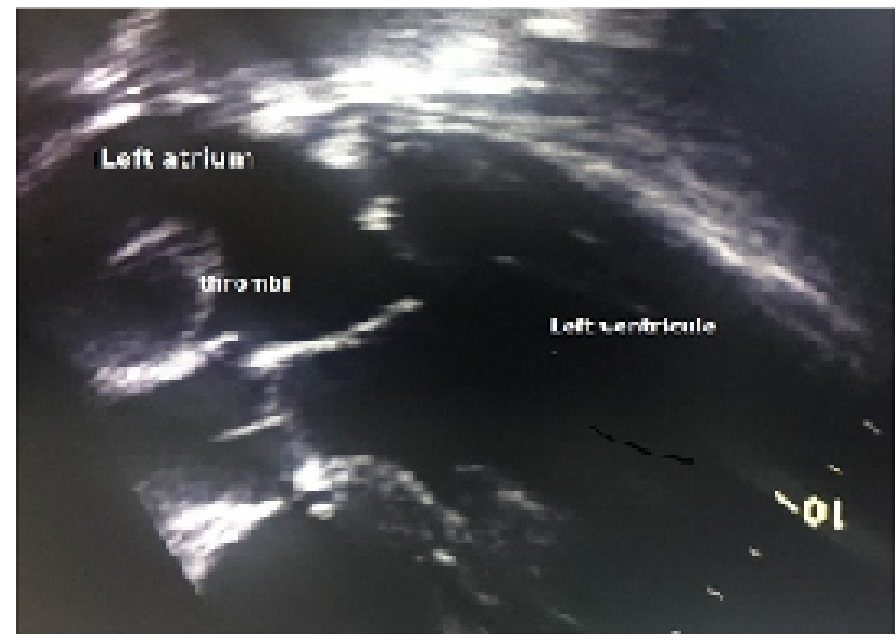

Figure 1. The echocardiographic image showing thrombus in left atrium

It is recommended to start treatment within 90-180 minutes for successful treatment and protection of kidney tissue. Other treatment options besides anticoagulant therapy are percutaneous transcatheter aspiration, embolectomy, and nephrectomy. Because of their high morbidity and limited renal tissue preservation, surgical treatment use is now very limited. Therefore, the risks and benefits are carefully evaluated, and conservative treatment should be considered for unilateral asymptomatic patients instead of surgical treatment $[2,3]$.

In conclusion, renal artery thrombosis is a rare clinical entity, and clinical findings are often nonspecific. So, the diagnosis is often delayed, which an important clinical condition is resulting in kidney parenchyma

${ }^{\star}$ Correspondence to: Erkut B, MD Professor, Department of Cardiovascular Surgery, Atatürk University, Medical Faculty, Erzurum, Turkey, Tel: 00905337451006; Email: bilgehanerkut@yahoo.com

Key words: renal artery, thrombosis, anticoagulant therapy, atrial fibrillation, acute abdominal pain

Received: May 21, 2019; Accepted: July 01, 2019; Published: July 04, 2019 


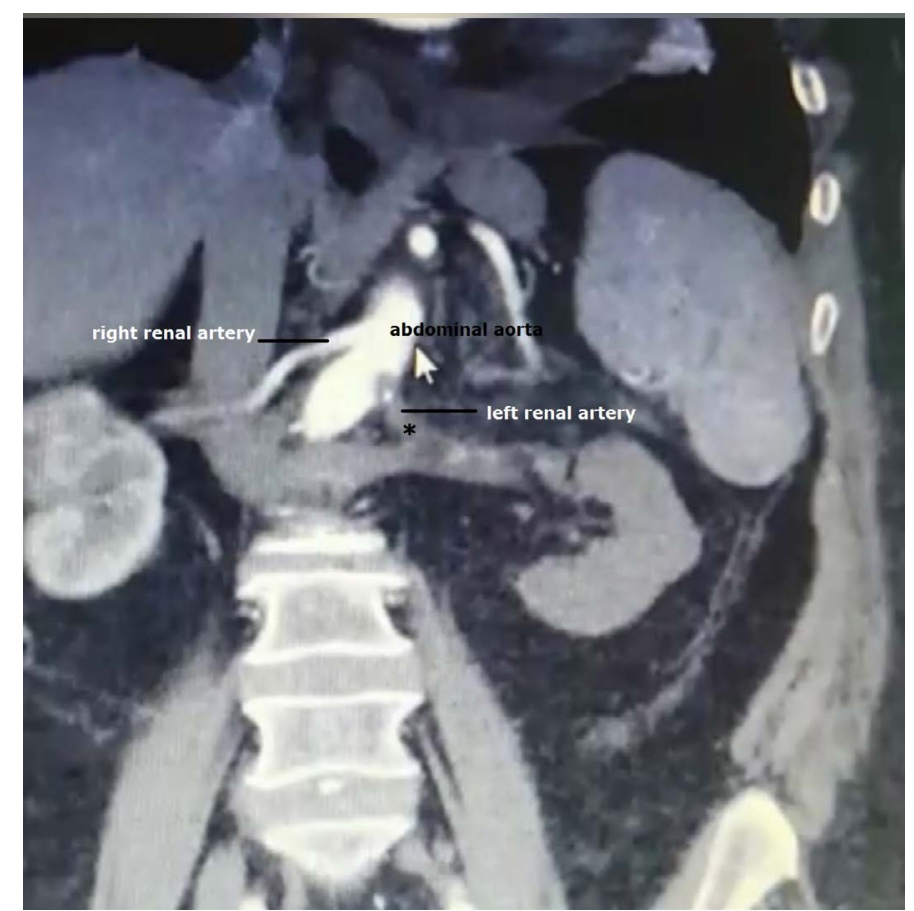

Figure 2. Sagittal view of arterial phase of computed tomography showing thrombi in left renal artery (black asterisk)

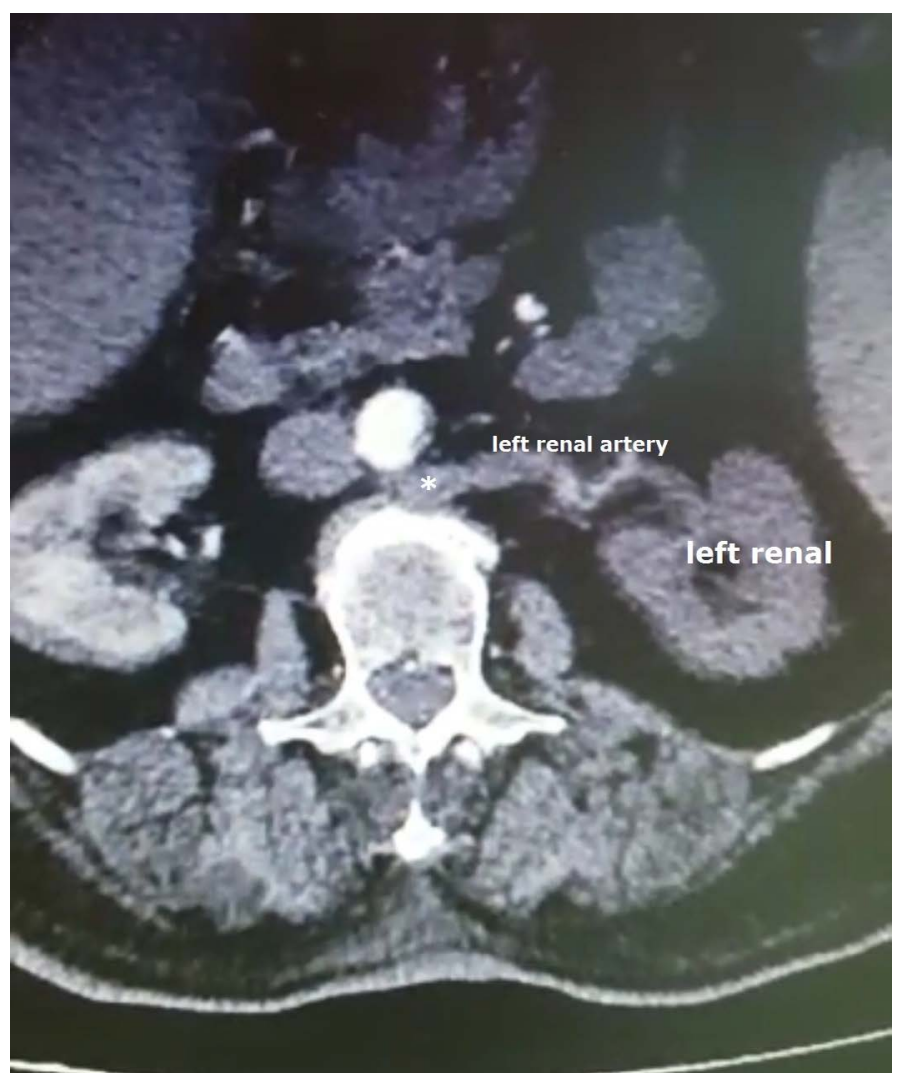

Figure 3. CT scan showing left renal artery thrombosis (white asterisk) and nonfunctional left renal 
loss. The purpose of this case report is to remind physicians that renal artery thrombosis should be considered in the differential diagnosis of abdominal pain complaints in patients admitted to hospitals and emergency departments. In cases with such complaints and with the suspicion of renal artery thrombosis, abdominal computed tomography is important for early diagnosis and kidney rescue.

\section{Competing Interests}

The authors declare that they have no competing interests, and did not receive funding for this article

\section{Consent}

Written informed consent was obtained from the patient's family for publication of this case report and any accompanying images.

\section{References}

1. Bourgault M, Grimbert P, Verret C, Pourrat J, Herody M, et al. (2013) Acute renal infarction: A case series. Clin J Am Soc Nephrol 8: 392-398.

2. Frost L, Engholm G, Johnsen S, Moller H, Henneberg EW, et al. (2001) Incident thromboembolism in the aorta and the renal, mesenteric, pelvic and extremity arteries after discharge from the hospital with a diagnosis of atrial fibrillation. Arch Intern Med 161: $272-276$

3. Domanovits H, Paulis M, Nikfardjam M, Meron G, Kürkciyan I, et al. (1999) Acute renal infarction. Clinical characteristics of 17 patients. Medicine (Baltimore) 78: 386-394.

Copyright: (2019 Erkut B. This is an open-access article distributed under the terms of the Creative Commons Attribution License, which permits unrestricted use, distribution, and reproduction in any medium, provided the original author and source are credited. 